\title{
IGR J18483-0311: an accreting X-ray pulsar observed by INTEGRAL
}

\author{
V. Sguera ${ }^{1}$, A. B. Hill ${ }^{1}$, A. J. Bird ${ }^{1}$, A. J. Dean ${ }^{1}$, A. Bazzano ${ }^{2}$, P. Ubertini ${ }^{2}$, N. Masetti ${ }^{3}$, R. Landi ${ }^{3}$, A. Malizia ${ }^{3}$, \\ D. J. Clark ${ }^{1}$, and M. Molina ${ }^{1}$
}

1 School of Physics and Astronomy, University of Southampton, Highfield, SO17 1BJ, UK e-mail: sguera@astro.soton.ac.uk

2 INAF/IASF Roma, via Fosso del Cavaliere 100, 00133 Roma, Italy

INAF/IASF Bologna, via Piero Gobetti 101, 40129 Bologna, Italy

Received 17 November 2006 / Accepted 10 February 2007

\section{ABSTRACT}

\begin{abstract}
Context. IGR J18483-0311 is a poorly known transient hard X-ray source discovered by INTEGRAL during observations of the Galactic Center region performed between 23-28 April 2003.

Aims. To detect new outbursts from IGR J18483-0311 using INTEGRAL and archival Swift XRT observations and finally to characterize the nature of this source using the optical/near-infrared (NIR) information available through catalogue searches.

Methods. We performed an analysis of light curves and spectra of INTEGRAL and archival Swift XRT data as well as of optical/NIR catalogues.

Results. We report on 5 newly discovered outbursts from IGR J18483-0311 detected by INTEGRAL. For two of them it was possible to constrain a duration of the order of a few days. The strongest outburst reached a peak flux of $\sim 120 \mathrm{mCrab}(20-100 \mathrm{keV})$; its broad band JEM-X/ISGRI spectrum (3-50 keV) is best fitted by an absorbed cutoff power law with $\Gamma=1.4 \pm 0.3$, cutoff energy of $22_{-45}^{+7.5} \mathrm{keV}$ and $N_{\mathrm{H}}=9_{-4}^{+5} \times 10^{22} \mathrm{~cm}^{-2}$. Timing analysis of INTEGRAL data allowed us to identify periodicities of 18.52 days and 21.0526 seconds which are likely the orbital period of the system and the spin period of the X-ray pulsar respectively. Swift XRT observations of IGR J18483-0311 provided a very accurate source position which strongly indicates a highly reddened star in the USNO-B1.0 and 2MASS catalogues as its possible optical/NIR counterpart.

Conclusions. The X-ray spectral shape, the periods of 18.52 days and $21.0526 \mathrm{~s}$, the high intrinsic absorption, the location in the direction of the Scutum spiral arm and the highly reddened optical object as possible counterpart, all favour the hypothesis that IGR J18483-0311 is a HMXB with a neutron star as compact companion. The system is most likely a Be X-ray binary, but a Supergiant Fast X-ray Transient nature can not be entirely excluded.
\end{abstract}

Key words. gamma rays: observations - X-rays: binaries - X-rays: bursts - X-rays: general - X-rays: individuals: IGR J18483-0311 - X-rays: binaries

\section{Introduction}

Since its launch in 2002, the INTEGRAL satellite has discovered more than one hundred new hard X-ray sources, mainly located toward the inner regions of the Galaxy which continue to be extensively monitored. Many of them are characterized by absorbed hard spectra, with little or no detectable emission in the soft X-rays since they are heavily absorbed by the interposing material. This, together with their often transient nature, explains why they have not been detected by any previous X-ray mission. As discussed by Dean et al. (2006), most of the newly discovered INTEGRAL sources should be high mass X-ray binaries (HMXBs), although Masetti et al. (2006a) found that several of them are actually Active Galactic Nuclei. This picture has been supported by various identifications with transient Be HMXBs or bright persistent highly absorbed supergiant HMXBs (SGXBs), either based on their secure identification at optical/infrared wavebands or on their X-ray characteristics and discovery of periodic pulsations (Walter et al. 2006). As well as the highly absorbed persistent SGXBs, INTEGRAL is also finding new members of a newly discovered class of SGXBs which escaped detection by previous X-ray missions mainly because of their fast X-ray transient behaviour. They have been labeled as Supergiant Fast X-ray Transients SFXTs (Negueruela et al. 2005; Sguera et al. 2005, 2006).

The transient X-ray source IGR J18483-0311 was discovered with the IBIS instrument (Ubertini et al. 2003) on board the INTEGRAL satellite during observations of the Galactic Center field performed between 23-28 April 2003 (Chernyakova et al. 2003). The average $\mathrm{X}$-ray flux was $\sim 10 \mathrm{mCrab}$ and $\sim 5 \mathrm{mCrab}$ in the energy bands $15-40 \mathrm{keV}$ and $40-100 \mathrm{keV}$ respectively. A possible X-ray outburst was observed on 26 April, when the X-ray flux increased up to $\sim 40 \mathrm{mCrab}(15-40 \mathrm{keV})$. Subsequently its discovery, Molkov et al. (2003a) reported another detection of the source by INTEGRAL on 5 April 2003. IGR J18483-0311 has also been detected by INTEGRAL at an average flux of $4.3 \pm 0.2 \mathrm{mCrab}(18-60 \mathrm{keV})$ during a survey of the Sagittarius arm tangent region in the spring 2003 (Molkov et al. 2003b). Stephen et al. (2006) reported the association between IGR J18483-0311 and the ROSAT HRI source 1RXH J184817.3-031017. Two optical USNO-B1.0 objects were found within the ROSAT positional uncertainty (Monet et al. 2003), one of which is also a near-infrared (NIR) 2MASS source (Skrutskie et al. 2006). 
Table 1. Summary of IBIS detections of newly discovered outbursts from IGR J18483-0311.

\begin{tabular}{cccccccc}
\hline \hline No. & Date & $\begin{array}{c}\text { Energy band } \\
(\mathrm{keV})\end{array}$ & $\begin{array}{c}\text { Peak flux } \\
(\mathrm{mCrab})\end{array}$ & $\begin{array}{c}\text { Peak luminosity } \\
\left(\mathrm{erg} \mathrm{s}^{-1}\right)\end{array}$ & $\begin{array}{c}\text { Duration } \\
(\text { days })\end{array}$ & $\begin{array}{c}\text { No. ScWs } \\
\text { Exp. time } \\
(\mathrm{ks})\end{array}$ \\
\hline 1 & 19 April 2006 & $20-100$ & $\sim 120$ & $\sim 7.8 \times 10^{36}$ & $\sim 1.8$ & 73 & 146.1 \\
2 & 5 September 2004 & $20-60$ & $\sim 95$ & $\sim 4.35 \times 10^{36}$ & $\sim 0.46^{\dagger}$ & 21 & 35.16 \\
3 & 26 April 2004 & $20-60$ & $\sim 80$ & & $\sim 3$ & 54 & 188.66 \\
4 & 18 March 2004 & $20-40$ & $\sim 135$ & & $\sim 0.33^{\dagger}$ & 16 & 28.29 \\
5 & 11 May 2003 & $20-60$ & $\sim 75$ & $\sim 3.2 \times 10^{36}$ & $\sim 1.3^{\dagger}$ & 50 & 104.05 \\
\hline
\end{tabular}

$\dagger$ Lower limit on the duration. ${ }^{\star}$ Assuming a distance of $\sim 5.7 \mathrm{kpc}$ (see Sect. 5).

In this paper, we report in Sect. 3.1 on IBIS detections of 5 newly discovered outbursts from IGR J18483-0311, not reported in the literature so far. Moreover, through timing analysis of the INTEGRAL light curves, we identify two periodicities which we interpret as being most likely attributable to the orbital period of the system (Sect. 3.2) and the spin period of an X-ray pulsar (Sect. 3.3). We report in Sect. 4 on two archival Swift XRT observations of IGR J18483-0311 which provide a very accurate source position. This allows us to pinpoint a highly reddened USNO-B1.0 star as the likely counterpart of the source, and its optical/NIR properties are discussed in Sect. 5.

\section{INTEGRAL data analysis}

IGR J18483-0311 was observed by JEM-X (Lund et al. 2003) and by IBIS/ISGRI (Lebrun et al. 2003). The reduction and analysis of the data have been performed using the INTEGRAL Offline Scientific Analysis (OSA) v.5.1. INTEGRAL observations are typically divided into short pointings (Science Windows, ScWs) of $\sim 2000$ s duration.

We performed an analysis at the $\mathrm{ScW}$ level of the deconvolved ISGRI shadowgrams searching for outburst activity from IGR J18483-0311. Our ScW data set consists of all Core Program observations (the Galactic Plane Survey and the Galactic Centre Deep Exposure) from revolution 45 (end of February 2003) to 430 (end of April 2006) as well as all public data released up to revolution 260 . The search provided 5 newly discovered outbursts from IGR J18483-0311. Due to possible cross-talk between objects in the same field of view (FOV), we have also investigated the variability pattern of all other bright sources in the FOV, in addition to that of the source of interest. They have shown a different time variability enabling us to conclude that the light curves obtained for the source of interest are reliable. Images from the X-ray monitor JEM-X were created for all newly discovered outbursts reported in this paper. Only in one case (outburst No. 1 in Table 1) was the source inside the JEM-X FOV so that it was possible to extract a spectrum and a $\mathrm{X}$-ray light curve.

\section{INTEGRAL results}

\subsection{IBIS detections of 5 newly discovered outbursts from IGR J18483-0311}

Table 1 lists the 5 newly discovered outbursts from IGR J18483-0311 detected by IBIS and discussed in the present paper. It provides the date and energy range of the detection, the flux and luminosity at the peak, the duration of the outburst activity, the number of ScWs during which the source is detected and the net exposure time.

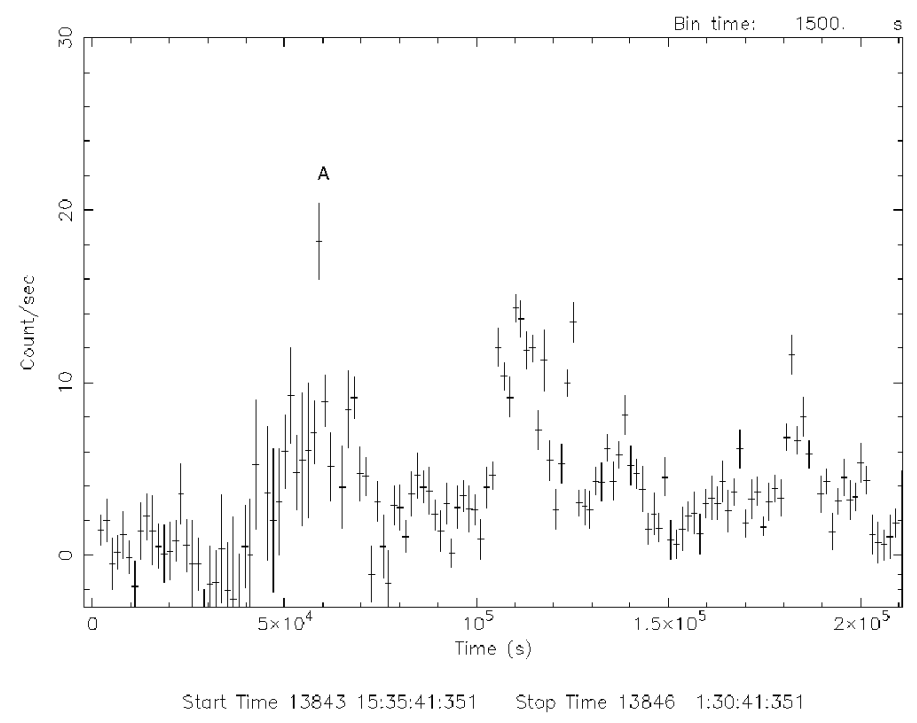

Fig. 1. ISGRI light curve (20-100 keV) of a newly discovered outburst of IGR J18483-0311 that occurred in April 2006 (No. 1 in Table 1). The strongest flare is labeled with the letter A.

Figure 1 shows the $20-100 \mathrm{keV}$ ISGRI light curve of outburst No. 1. At the beginning there is no significant detection since the count rate is consistent with zero count/sec, then suddenly on 19 April $2006 \sim 04: 50$ UTC the source turned on. Initially, the outburst activity was characterized by a prominent flare (labeled as A in Fig. 1) which reached a peak flux of $\sim 120 \mathrm{mCrab}$ or $2.04 \times 10^{-9} \mathrm{erg} \mathrm{cm}^{-2} \mathrm{~s}^{-1}(20-100 \mathrm{keV})$ in a few hours and then dropped with the same timescale. This first flare was followed by several others, then the source turned off. The duration of the total outburst activity was $\sim 1.8$ days.

We combined all the ScWs during outburst No. 1 into a single mosaic significance map. IGR J18483-0311 was detected at $\sim 50 \sigma(20-100 \mathrm{keV})$, and its coordinates $(\mathrm{J} 2000)$ are RA = $18^{\mathrm{h}} 48^{\mathrm{m}} 15.07^{\mathrm{s}}$, Dec $=-03^{\circ} 10^{\prime} 17^{\prime \prime}$, with an error radius of 1'.4.

The combined JEM-X/ISGRI spectrum (3-50 keV) from the whole duration of the outburst No. 1 is best fitted by an absorbed cutoff power law $\left(\chi_{v}^{2}=1.19,140\right.$ d.o.f. $)$ with $\Gamma=$ $1.4 \pm 0.3, N_{\mathrm{H}}=9_{-4}^{+5} \times 10^{22} \mathrm{~cm}^{-2}$ and cutoff energy equal to $22_{-4.5}^{+7.5} \mathrm{keV}$. The absorption exceeds the galactic one along the line of sight $\left(1.6 \times 10^{22} \mathrm{~cm}^{-2}\right)$, suggesting that most of the low energy absorption is intrinsic to the source. Figure 2 displays the absorbed cutoff power law unfolded spectrum, while Fig. 3 shows the contour plot for the photon index and the cutoff energy. To account for a cross-calibration mismatch between the two instruments we have introduced a constant in the fit, which when left free to vary provides a value of $1.3 \pm 0.15$. It is worth pointing out that a similarly good fit $\left(\chi_{v}^{2}=1.2,141\right.$ 


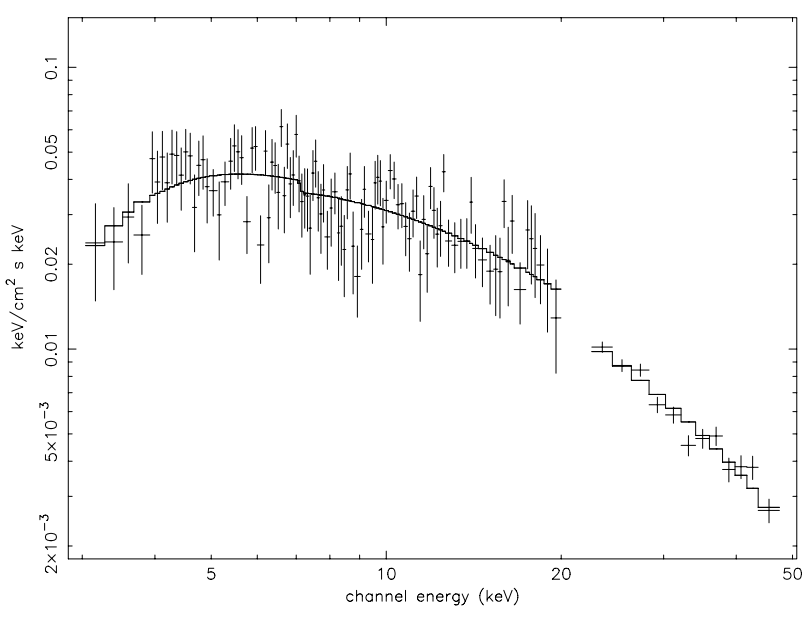

Fig. 2. Unfolded combined JEM-X and ISGRI spectrum (3-50 keV) of IGR J18483-0311 during outburst No. 1 in Table 1.

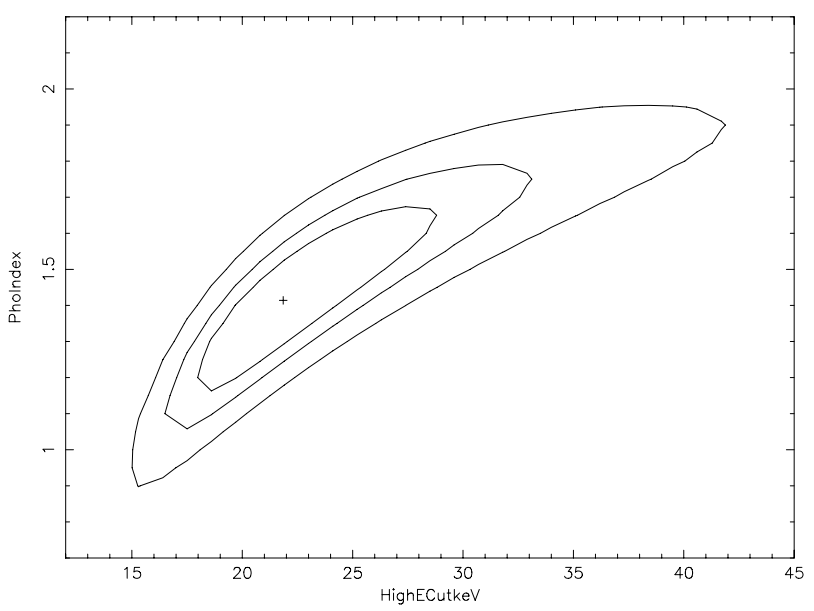

Fig. 3. Confidence contours level for the photon index and the cutoff energy from the spectral analysis of the outburst No. 1 in Table 1.

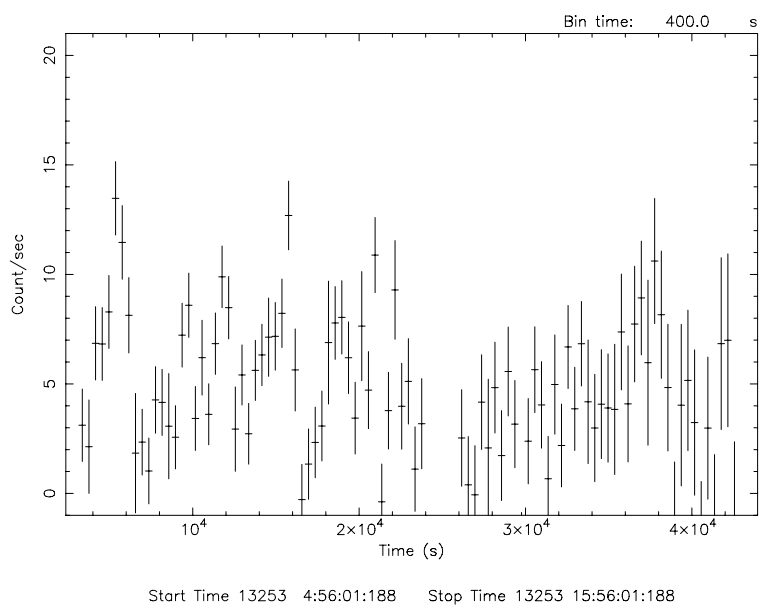

Fig. 4. ISGRI light curve (20-60 keV) of a newly discovered outburst of IGR J18483-0311 that occurred in September 2004 (No. 2 in Table 1).

d.o.f.) is also provided by an absorbed bremsstrahlung model with $k T=21.5_{-2}^{+2.5} \mathrm{keV}$ and $N_{\mathrm{H}}=7.5_{-2}^{+2.5} \times 10^{22} \mathrm{~cm}^{-2}$. The latter is compatible, within the uncertainties, with the value obtained from the absorbed cutoff power law spectral model.

Figure 4 shows the 20-60 keV ISGRI light curve of outburst No. 2 that started on 5 September 2004 at 04:50 UTC

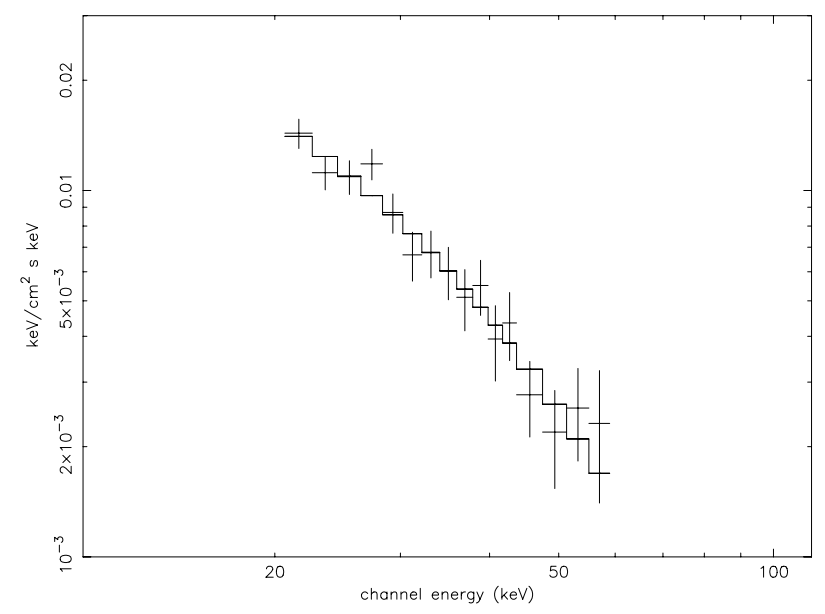

Fig. 5. Unfolded bremsstrahlung spectrum $(20-60 \quad \mathrm{keV})$ of IGR J18483-0311 during outburst No. 2 in Table 1.

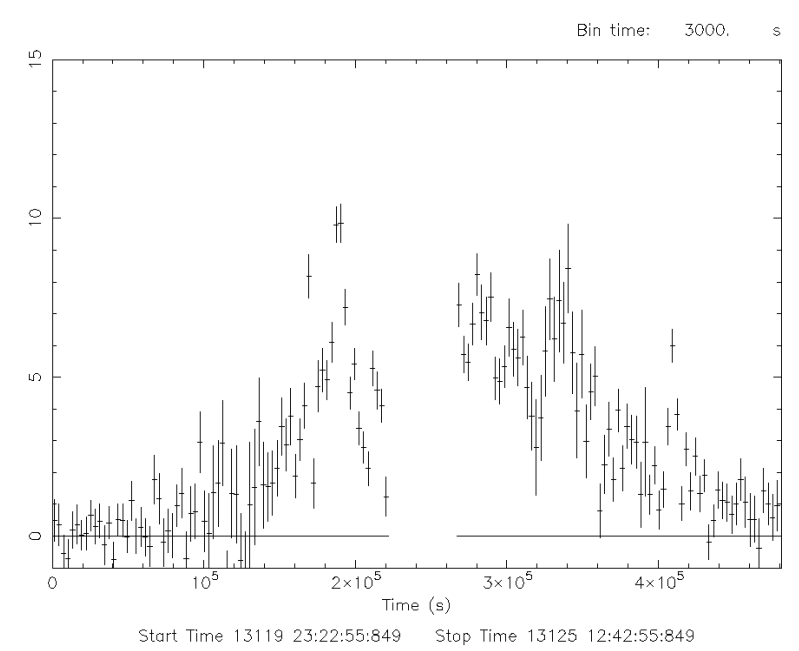

Fig. 6. ISGRI light curve (20-60 keV) of a newly discovered outburst of IGR J18483-0311 that occurred in April 2004 (No. 3 in Table 1).

and ended on the same day at $\sim 16: 00$ UTC. It is truncated at the beginning and at the end because the source was outside the IBIS FOV, so that it is not possible to constrain a total duration for the outburst activity. The source was in the IBIS FOV for $\sim 11 \mathrm{~h}$ and its light curve is characterized by several fast flares (on timescales of few tens of minutes) reaching a maximum peak flux $(20-60 \mathrm{keV})$ of $\sim 95 \mathrm{mCrab}$ or $\sim 1.1 \times 10^{-9} \mathrm{erg} \mathrm{cm}^{-2} \mathrm{~s}^{-1}$. The 20-60 keV spectrum extracted during the whole outburst is well fitted by thermal models such as black body $\left(\chi_{v}^{2}=0.97,14\right.$ d.o.f., $\left.k T=6.5_{-0.45}^{+0.45} \mathrm{keV}\right)$ or bremsstrahlung $\left(\chi_{v}^{2}=0.61,14\right.$ d.o.f., $\left.k T=20.6_{-3}^{+4} \mathrm{keV}\right)$. A good fit is also provided by a simple power law with $\Gamma=2.9_{-0.25}^{+0.25}\left(\chi_{v}^{2}=0.74,14\right.$ d.o.f. $)$. Figure 5 shows the unfolded 20-60 keV bremsstrahlung spectrum.

Figure 6 displays the 20-60 keV light curve of outburst No. 3 in Table 1 . The gap of $\sim 13 \mathrm{~h}$ is due to the visibility constraint between one INTEGRAL revolution and the next. In spite of that, the flaring activity of the source is very evident. Initially the flux is consistent with zero, then suddenly the source turned on at 20:00 UTC on 26 April 2004 and reached a maximum peak flux of $\sim 80 \mathrm{mCrab}(20-60 \mathrm{keV})$; the duration of the total outburst activity was $\sim 3$ days. A spectrum extracted during the whole outburst $(20-60 \mathrm{keV})$ is equally well fitted by a black body $\left(\chi_{v}^{2}=0.95,14\right.$ d.o.f., $\left.k T=7.2_{-0.25}^{+0.25} \mathrm{keV}\right)$ or bremsstrahlung $\left(\chi_{v}^{2}=0.95,14\right.$ d.o.f., $\left.k T=25.2_{-2.2}^{+2.5} \mathrm{keV}\right)$. A simple power law 


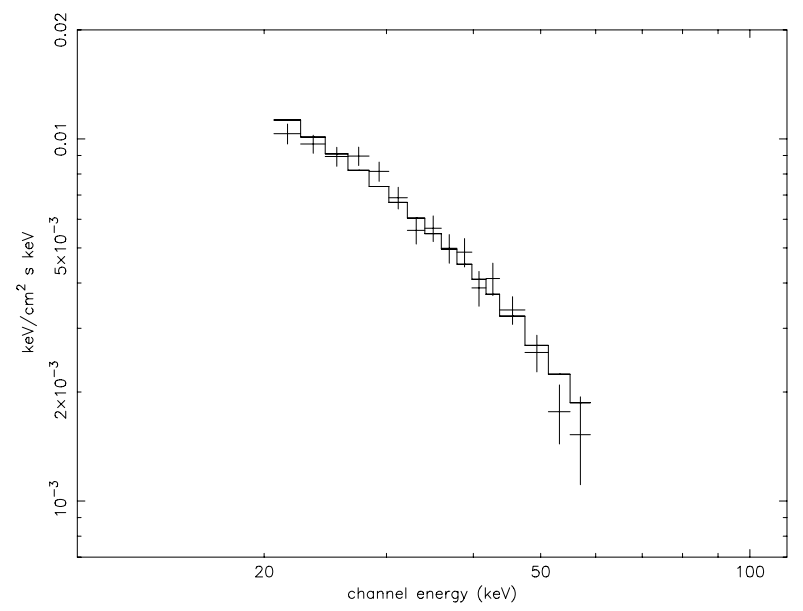

Fig. 7. Unfolded bremsstrahlung spectrum of outburst No. 3 in Table 1.

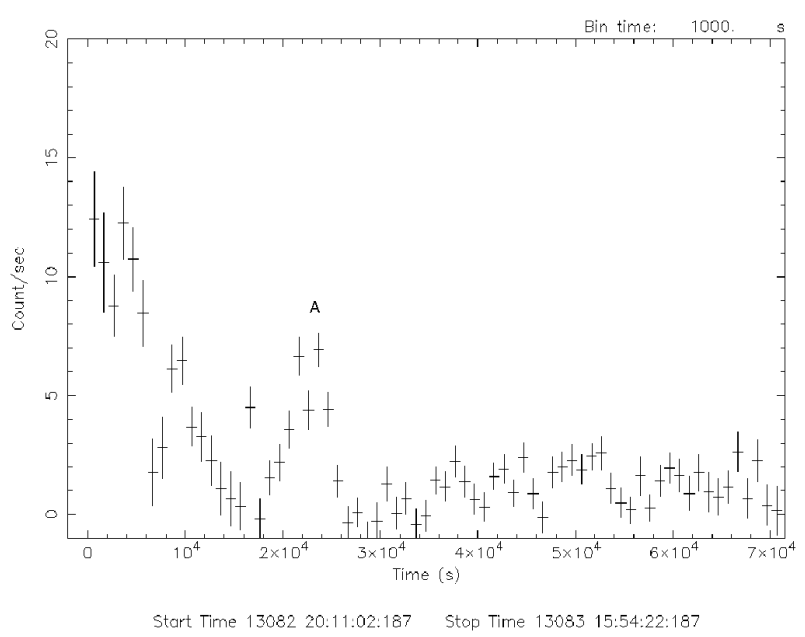

Fig. 8. ISGRI light curve (20-40 keV) of a newly discovered outburst of IGR J18483-0311 that occurred in March 2004 (No. 4 in Table 1).

provides a bad fit $\left(\chi_{v}^{2}=2.05,14\right.$ d.o.f.). Figure 7 shows the unfolded bremsstrahlung spectrum.

The ISGRI light curve (20-40 keV) of outburst No. 4 is shown in Fig. 8. The observations starts on 18 March 2004 at 20:00 The beginning was not observed because the source was outside the IBIS FOV. Nevertheless, the decay of the flare is clear, in fact the 20-40 keV flux dropped from $\sim 135 \mathrm{mCrab}$ $\left(\sim 1.02 \times 10^{-9} \mathrm{erg} \mathrm{cm}^{-2} \mathrm{~s}^{-1}\right)$ to just a few mCrab in $\sim 4 \mathrm{~h}$. This strongly suggests that the source was active before entering the IBIS FOV. Another flare is present in the light curve (labeled as A): it reached a peak flux of $\sim 80 \mathrm{mCrab}(20-40 \mathrm{keV})$ in $\sim 1 \mathrm{~h}$ and then dropped to an almost null flux with the same timescale. The remaining part of the light curve shows no flaring activity from the source. The $20-60 \mathrm{keV}$ spectrum extracted during its whole outburst cannot be fitted by a simple power law $\left(\chi_{v}^{2}=1.59\right.$, 14 d.o.f.). Reasonable fits were obtained by thermal models such as black body $\left(\chi_{v}^{2}=0.85,14\right.$ d.o.f., $\left.k T=7.8_{-0.6}^{+0.6} \mathrm{keV}\right)$ or bremsstrahlung $\left(\chi_{v}^{2}=1.26,14\right.$ d.o.f., $\left.k T=32.5_{-6.7}^{+10.5} \mathrm{keV}\right)$. Figure 9 shows the unfolded bremsstrahlung spectrum.

Finally, Fig. 10 shows the 20-60 keV ISGRI light curve of outburst No. 5 in Table 1. It started on 11 May 2003 at $\sim$ 19:30 UTC and it ended on 13 May 2003 at $\sim 02: 30$ UTC. It is truncated at the beginning and at the end because the source was outside the IBIS FOV, so it is not possible to constrain a total duration of the outburst. The peak flux was $\sim 75 \mathrm{mCrab}$

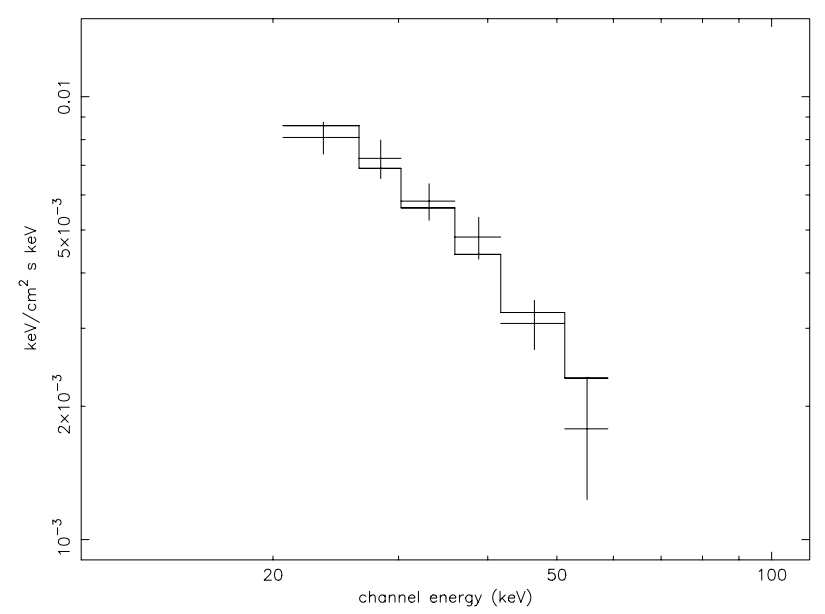

Fig. 9. Unfolded bremsstrahlung spectrum of outburst No. 4 in Table 1.

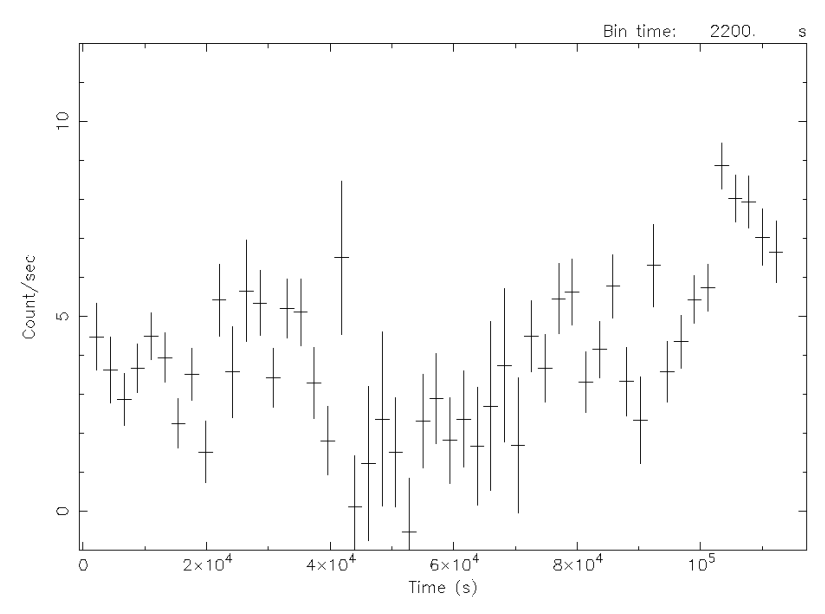

Start Time 12770 19:37:09:949 Stop Time $12772 \quad$ 2:47:09:949

Fig. 10. ISGRI light curve (20-60 keV) of a newly discovered outburst of IGR J18483-0311 that occurred in May 2003 (No. 5 in Table 1).

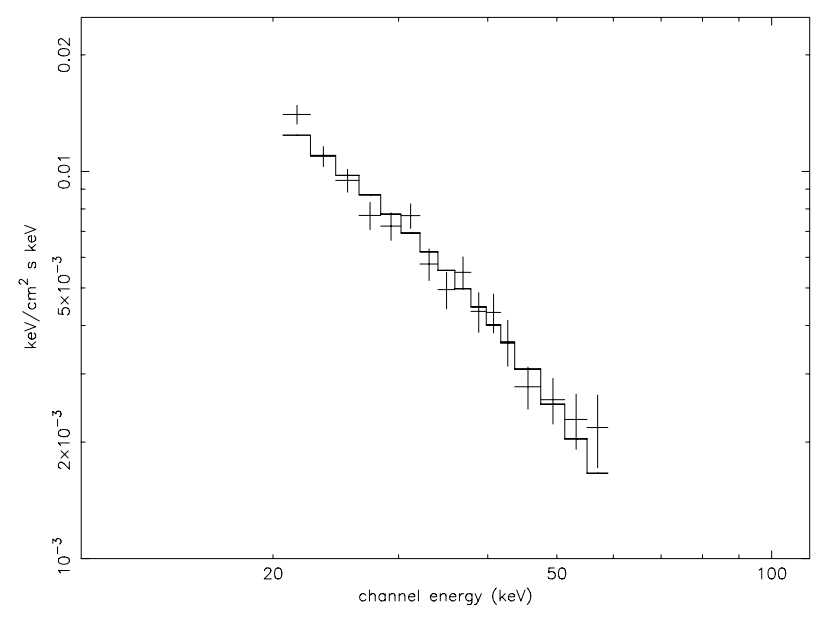

Fig. 11. Unfolded bremsstrahlung spectrum (20-60 keV) of outburst No. 5 in Table 1 of IGR J18483-0311.

(20-60 keV). The 20-60 keV spectrum of the whole outburst is best fitted by a bremsstrahlung model $\left(\chi_{v}^{2}=1.1,14\right.$ d.o.f. $)$ with $k T=22_{-2.5}^{+2} \mathrm{keV}$, whereas a black body is a very bad description to the data $\left(\chi_{v}^{2}=3.2,14\right.$ d.o.f. $)$. A reasonable fit is also provided by a simple power law $\left(\chi_{v}^{2}=0.85,14\right.$ d.o.f. $)$ with $\Gamma=2.9_{-0.15}^{+0.15}$. 
Table 2. Summary of spectral analysis of the 5 newly discovered outbursts from IGR J18483-0311. The name of the spectral models are written in XSPEC terminology.

\begin{tabular}{|c|c|c|c|c|c|c|}
\hline Model & Parameter & $\begin{array}{c}\text { Outburst No. } 1 \\
(3-50 \mathrm{keV})\end{array}$ & $\begin{array}{c}\text { Outburst No. } 2 \\
(20-60 \mathrm{keV})\end{array}$ & $\begin{array}{l}\text { Outburst No. } 3 \\
(20-60 \mathrm{keV})\end{array}$ & $\begin{array}{c}\text { Outburst No. } 4 \\
(20-60 \mathrm{keV})\end{array}$ & $\begin{array}{c}\text { Outburst No. } 5 \\
(20-60 \mathrm{keV})\end{array}$ \\
\hline \multirow[t]{4}{*}{ wa+cutoffpl } & $N_{\mathrm{H}}\left(10^{22} \mathrm{~cm}^{-2}\right)$ & $9_{-4}^{+5}$ & & & & \\
\hline & $\Gamma$ & $1.4 \pm 0.3$ & & & & \\
\hline & $E_{\mathrm{c}}$ & $22_{-4.5}^{+7.5}$ & & & & \\
\hline & $\chi_{v}^{2}$ (d.o.f.) & $1.19(140)$ & & & & \\
\hline \multirow[t]{3}{*}{ wa+bremss } & $N_{\mathrm{H}}\left(10^{22} \mathrm{~cm}^{-2}\right)$ & $7.5_{-2}^{+2.5}$ & & & & \\
\hline & $k T$ & $21.5_{-2}^{+2.5}$ & & & & \\
\hline & $\chi_{v}^{2}$ (d.o.f.) & $1.2(141)$ & & & & \\
\hline \multirow[t]{2}{*}{ bremss } & $k T$ & $26_{-2.5}^{+3.3}$ & $20.6_{-3}^{+4}$ & $25.2_{-2.2}^{+2.5}$ & $32.5_{-6.7}^{+10.5}$ & $22_{-2.5}^{+2}$ \\
\hline & $\chi_{v}^{2}$ (d.o.f.) & $1.49(142)$ & $0.61(14)$ & $0.95(14)$ & $1.26(14)$ & $1.1(14)$ \\
\hline \multirow[t]{2}{*}{$\overline{b b}$} & $k T$ & & $6.5_{-0.45}^{+0.45}$ & $7.2_{-0.25}^{+0.25}$ & $7.8_{-0.6}^{+0.6}$ & \\
\hline & $\chi_{v}^{2}$ (d.o.f.) & $4.86(142)$ & $0.97(14)$ & $0.95(14)$ & $0.85(14)$ & $3.23(14)$ \\
\hline \multirow[t]{2}{*}{ po } & $\bar{\Gamma}$ & & $2.9_{-0.25}^{+0.25}$ & & & $2.9_{-0.15}^{+0.15}$ \\
\hline & $\chi_{v}^{2}$ (d.o.f.) & $2.65(142)$ & $0.74(14)$ & $2.05(14)$ & $1.59(14)$ & $0.85(14)$ \\
\hline
\end{tabular}

Figure 11 shows the unfolded $20-60 \mathrm{keV}$ bremsstrahlung spectrum.

Table 2 is a summary of the spectral analysis of each newly discovered outburst. Outburst No. 1 is the only one for which a broad band spectrum (3-50 keV) is available. The cutoff power law model constrains very well its spectral properties both at soft and hard X-rays, with best fit parameters $\Gamma=1.4 \pm 0.3, E_{\mathrm{c}}=$ $22_{-4.5}^{+7.5} \mathrm{keV}$ and $N_{\mathrm{H}}=9_{-4}^{+5} \times 10^{22} \mathrm{~cm}^{-2}$. Moreover, it can be noted that a bremsstrahlung model provided a good description of all 5 outbursts with a similar temperature $k T$ in the range $20-32 \mathrm{keV}$. A black body gave good fits for the outbursts Nos. 2, 3 and 4 with a similar temperature $k T$ in the range $6.5-7.5 \mathrm{keV}$, but is unacceptable in the case of the outbursts Nos. 1 and 5. Finally, a simple power law described well the spectra of outburst No. 2 and 5 providing the same value of the photon index ( 2.9) Whereas, outbursts Nos. 3 and 4 could not be described by a simple power law.

\subsection{Recurrence timescale}

We investigated the long term light curve of IGR J18483-0311 searching for any evidence of periodicity which would indicate the recurrence timescale of its transient activity. The flux of IGR J18483-0311 was extracted from each ISGRI pointing where the source was within $12^{\circ}$ of the centre of the field of view, producing a long term light curve of the source on the $\mathrm{ScW}$ timescale. A $12^{\circ}$ limit was applied because the off-axis response of ISGRI is not well modelled at large off-axis angles and consequently in combination with the telescope dithering (or the movement of the source within the FOV) introduces a systematic error in the measurement of source fluxes generating problems in the detection of periodic signals (Hill 2006). The 20-40 keV light curve was then searched for periodicities using the Lomb-Scargle periodogram method by means of the fast implementation of Press \& Rybicki (1989) and Scargle (1982). The resulting power spectrum is shown in Fig. 12; the peak power of 165.7 corresponds to a frequency of 0.0540 days $^{-1}$. This frequency equates to a period of 18.52 days with a theoretical Lomb-Scargle error of \pm 0.01 days. The error on the angular frequency is given by Horne \& Baliunas (1986).

$\delta \omega=\frac{3 \pi \sigma_{\mathrm{N}}}{2 \sqrt{N} T A}$

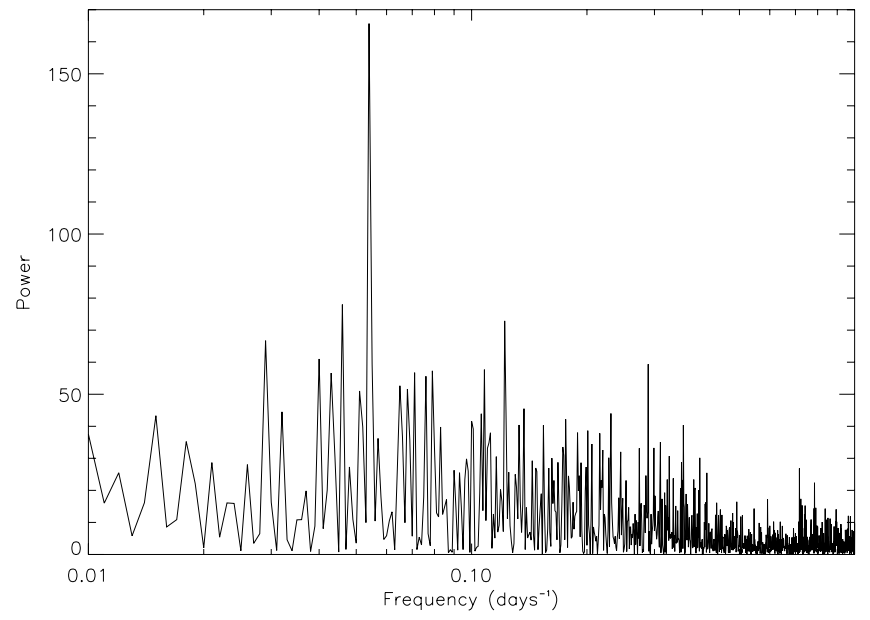

Fig. 12. Lomb-Scargle periodogram generated from the $20-40 \mathrm{keV}$ light curve of IGR J18483-0311.

where $\sigma_{\mathrm{N}}^{2}$ is the variance of the noise, $N$ is the number of data points, $T$ is the total length of the data set and $A$ is the amplitude of the signal given by:

$A=2 \sqrt{\frac{z_{0} \sigma_{s}^{2}}{N}}$

where $z_{0}$ is the Lomb-Scargle power and $\sigma_{s}^{2}$ is the variance of the light curve. The period estimate was confirmed with a MonteCarlo simulation. Each flux measurement was adjusted using Gaussian statistics within its individual error estimate to generate a simulated light curve of the source. The corresponding periodogram was produced and any detected periodicity recorded. We simulated 200000 light curves in this fashion and found that $99.5 \%$ identified a periodicity centred at 18.52 days. The phasefolded 20-40 keV lightcurve of IGR J18483-0311 is shown in Fig. 13. A clear flare-like profile is apparent, with the source being predominantly undetected and then briefly flaring to a detectable level. The average duration of an outburst can be seen to be of the order of 3 days (FWHM). This is consisted with the measured durations for outburst Nos. 1 and 3 in Table 1. 
Table 3. Analysis of the $20-40 \mathrm{keV}$ light curve of IGR J18483-0311 around the expected start time of outbursts based upon the 18.52 days periodicity. For the outburst numbers not listed there was no significant coverage of the source by INTEGRAL around those times.

\begin{tabular}{llll}
\hline \hline Outburst No. & Expected start time (MJD) & INTEGRAL coverage (MJD) & Comments \\
\hline 1 & $\sim 52714.48$ & $52714.99-52715.63$ & Low level of outburst activity (maximum peak flux 20 mCrab) \\
2 & $\sim 52733$ & $52734.92-52735.61$ & Outburst reported by Molkov et al. 2003a (peak flux 40 mCrab) \\
3 & $\sim 52751.52$ & $52753.04-52757.50$ & Outburst when the source was discovered (Chernyakova et al. 2003) \\
4 & $\sim 52770.04$ & $52770.80-52772.08$ & Outburst discussed in this paper (No. 5 in Table 1) \\
21 & $\sim 53084.88$ & $53082.85-53083.62$ & Outburst discussed in this paper (No. 4 in Table 1) \\
23 & $\sim 53121.92$ & $\sim 53121.7^{\star}$ & Outburst discussed in this paper (No. 3 in Table 1) \\
30 & $\sim 53251.56$ & $53253.20-53253.60$ & Outburst discussed in this paper (No. 2 in Table 1) \\
62 & & $\sim 53844.2^{\star}$ & Outburst discussed in this paper (No. 1 in Table 1) \\
\hline
\end{tabular}

* Start time of the outburst.

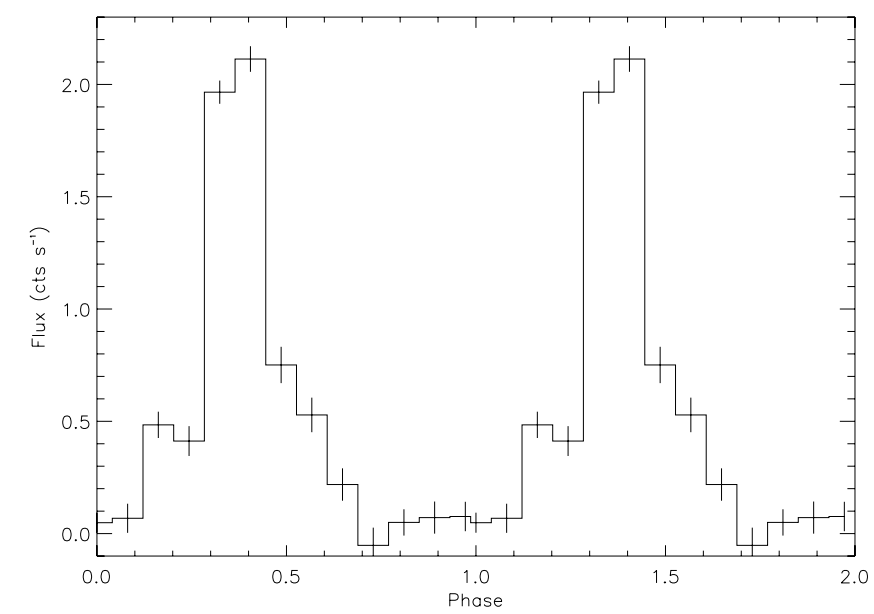

Fig. 13. The phase-folded background subtracted $20-40 \mathrm{keV}$ light curve of IGR J18483-0311. The data are folded on a period of 18.52 days.

\subsubsection{Looking for missed outbursts}

The detected periodicity of $\sim 18.52$ days indicates the recurrence timescale of outbursts from IGR J18483-0311, so that we could search for additional weaker outbursts in the INTEGRAL observations. Between the first and last observation analysed in this paper (52 704-53846 MJD) 62 outbursts may have occurred. Using the measured start time (MJD 53 844.2) of outburst No. 1 in Table 1 and the periodicity of 18.52 days, the full long term light curve of IGR J18483-0311 was examined around the predicted outburst start times. To this aim, no off-axis angle limit was applied during the extraction of full long term ISGRI light curve. The results of this examination are shown in Table 3 . Of the 62 potential outbursts, only 8 were well covered by INTEGRAL observations. For the outburst numbers not listed there was no significant coverage of the source by INTEGRAL around those times. These 8 outbursts include the 5 reported brightest ones in Table 1 and discussed in this paper, 2 outbursts with a low level of activity and the outburst when the source was discovered by Chernyakova et al. (2003). It should be noted that all but one of the outbursts listed in Table 3 occured when expected as based upon the 18.52 days periodicity. The only exception (No. 21 in Table 3 or No. 4 in Table 1) occured at least 2 days earlier than expected. This may in part be explained by the difficulty in identifying a period when each outburst has a unique shape, moreover only 2 out of 5 outbursts detected by IBIS have been seen for their entire duration. Consequently the statistical error assigned to the 18.52 days period is likely to be underestimate. However, it might also indicate that the outbursting behaviour of the source is only semi-regular, implying that

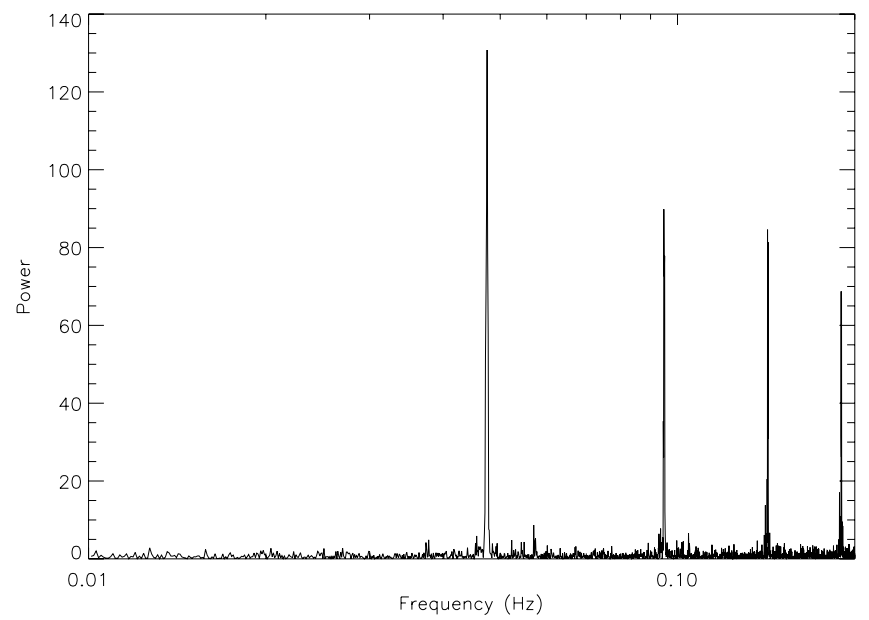

Fig. 14. Lomb-Scargle periodogram generated from the JEM-X 4-20 keV light curve of IGR J18483-0311 during the outburst No. 1 in Table 1 . The periodicity at $0.04750 \mathrm{~Hz}$ is clearly apparent as are the 1st, 2nd and 3rd harmonics.

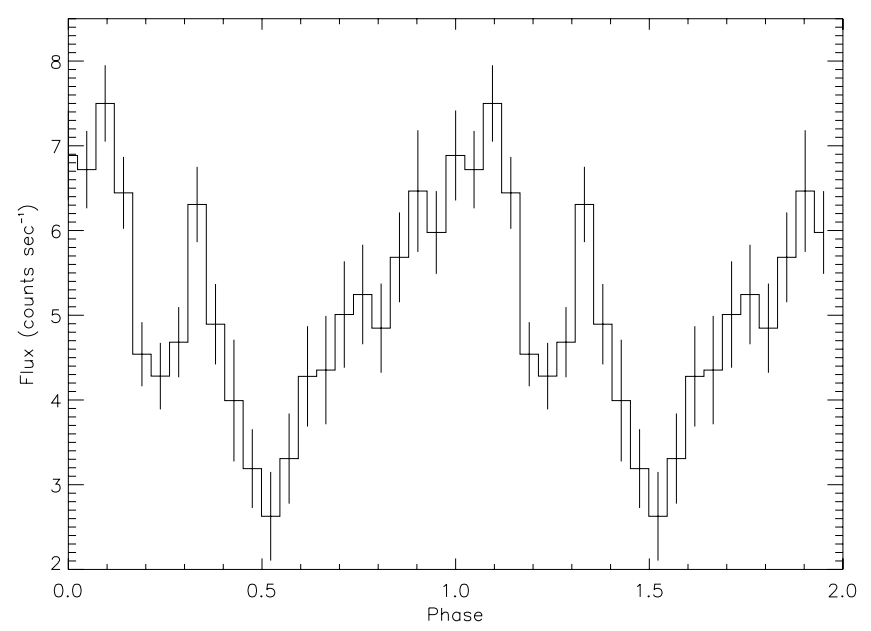

Fig. 15. The phase-folded 4-20 keV light curve of IGR J18483-0311. The data are folded on the detected period of $21.0526 \mathrm{~s}$.

there may be something which modulates the outburst time scale beyond the mechanism that produces the periodicity. This behaviour could be similar to that observed in Be X-ray transients in which the outbursts are associated with both the orbital period of the system and with the size of the disk around the Be star. 


\subsection{Searching for a pulse period}

Fine timing resolution ISGRI light curves $(20-40 \mathrm{keV})$ were constructed for the duration of the two brightest outbursts (Nos. 1 and 4) in Table 1, using the ii-light tool in OSA5.1. A search for pulsations was performed using the Lomb-Scargle periodogram method with no positive results.

However, IGR J18483-0311 was also inside the JEM-X FOV during part of the outburst No. 1 in Table 1 . Consequently, we produced a light curve in the 4-20 keV band using the standard OSA5.1 tools. This light curve was also searched for periodicities using the Lomb-Scargle method; the resulting power spectrum is shown in Fig. 14. The peak power of 130.8 corresponds to a frequency of $0.04750 \mathrm{~Hz}$. The error on this measurement is calculated using Eqs. (1) and (2). The corresponding period is $21.0526 \pm 0.0005$ seconds; the $1 \mathrm{st}$, 2nd and $3 \mathrm{rd}$ harmonics of this period are also evident in the power spectrum.

Folding the JEM-X light curve on the $21.0526 \mathrm{~s}$ yields the pulse-period phase-folded light curve shown in Fig. 15. We attribute this periodicity to the spin period of an X-ray pulsar located within the system. There are no significantly detected features in the profile. The pulse fraction, $\left(I_{\max }-I_{\min }\right) / I_{\max }$, where $I_{\max }$ and $I_{\min }$ are the maximum and minimum count rates respectively, is $\sim(65 \pm 10) \%$.

\section{Swift observations and results}

In this section we report on X-ray observations acquired with the XRT (X-ray Telescope) on board the Swift satellite (Gehrels et al. 2004). A search of the XRT data archive revealed that Swift carried out 2 observations of IGR J18483-0311, on 16 February 2006 and 5 March 2006. The XRT collected data for a total exposure time of $8 \mathrm{ks}$ and $5.6 \mathrm{ks}$, respectively. Unfortunately in both observations the source was outside the IBIS FOV. XRT data reduction was performed using XRTDAS v. 2.4 standard data pipeline package (XRTPIPELINE v. 0.10.3), in order to produce screened event files. All data are extracted only in the Photon Counting (PC) mode, adopting the standard grade filtering (0-12 for PC) according to the XRT nomenclature. Events for spectral analysis were extracted within a circular region of radius $20^{\prime \prime}$, which encloses about $90 \%$ of the PSF at $1.5 \mathrm{keV}$ (Moretti et al. 2004), centered on the source position. The background was extracted from various source-free regions close to the $\mathrm{X}$ ray source of interest using both circular/annular regions with different radii, in order to ensure an evenly sampled background.

First of all, the Swift XRT analysis of IGR J18483-0311 provided a very accurate position $\left(\mathrm{RA}=18^{\mathrm{h}} 48^{\mathrm{m}} 17.17^{\mathrm{s}}\right.$, Dec $=$ $-03^{\circ} 10^{\prime} 15.54^{\prime \prime}, \mathrm{J} 2000$ ) with the error radius being equal to $3^{\prime \prime} .3$. This refined source position was obtained using the method reported in Moretti et al. (2006), which allows positional errors accurate to $3^{\prime \prime}$.

For the observation of 16 February 2006 (OBS1), it was possible to extract a meaningful spectrum only in the energy range 1-7 keV. Above $7 \mathrm{keV}$ and below $1 \mathrm{keV}$ the statistics were not good enough to perform a spectral analysis. The spectrum from $1-7 \mathrm{keV}$ is best fitted by an absorbed power law $\left(\chi_{v}^{2}=1.38\right.$, 38 d.o.f.) with $\Gamma=1.7 \pm 0.7$ and $N_{\mathrm{H}}=6.3_{-1.8}^{+2.4} \times 10^{22} \mathrm{~cm}^{-2}$. Thermal models such as black body or bremsstrahlung provide very bad fits with a $\chi_{v}^{2}$ greater than 2 . The spectrum $(1-10 \mathrm{keV})$ extracted during the observation of 5 March 2006 (OBS2) is best fitted by an absorbed power law $\left(\chi_{v}^{2}=1.009,67\right.$ d.o.f.) with $\Gamma=1.3 \pm 0.25$ and $N_{\mathrm{H}}=4.8 \pm 0.9 \times 10^{22} \mathrm{~cm}^{-2}$. Thermal models such as black body or bremsstrahlung are again a bad description of the data. Table 4 provides a list of the characteristics of

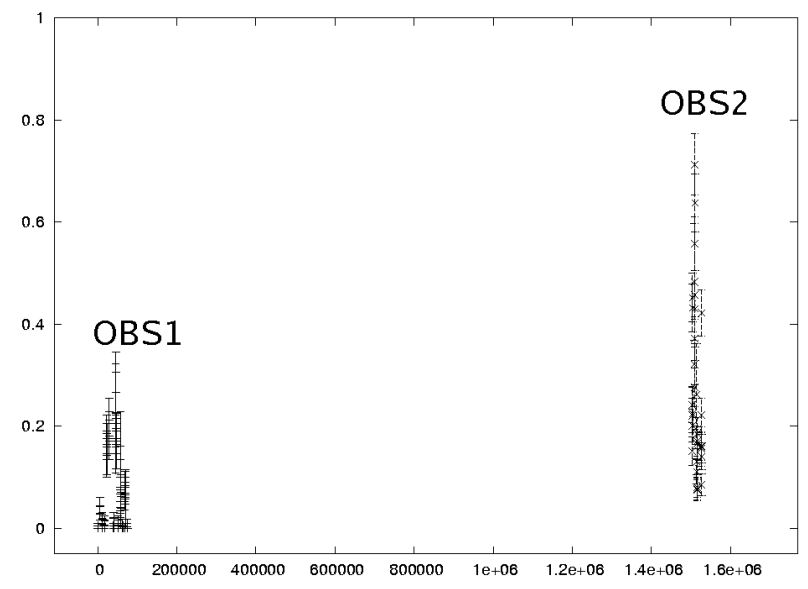

Fig. 16. Swift XRT light curve $(0.2-10 \mathrm{keV})$ light curve of the two Swift XRT observations. The bin time is 200 s. Time axis is in seconds, counted from the beginning of the first Swift XRT observation. Flux axis is in count/s.

the two Swift XRT observations of IGR J18483-0311. In order to check for variability in the spectral index between the two Swift observations, both absorbed power law spectra have been fitted fixing the $N_{\mathrm{H}}$ to the average value found from the previous spectral analysis $\left(N_{\mathrm{H}}=5.55 \times 10^{22} \mathrm{~cm}^{-2}\right)$. By doing so, no variability has been found since the photon index assumed an identical value of $1.5 \pm 0.25$ (OBS1) and $1.5 \pm 0.13$ (OBS2), respectively.

Taking into account the Swift XRT spectra of IGR J18483-0311, we extrapolated its flux in the ROSAT energy range $0.1-2 \mathrm{keV}$. A count rate of $\sim 0.00046 \mathrm{count} / \mathrm{s}$ was obtained, which is $\sim 30$ times smaller than that of the ROSAT HRI source (0.0139 count/s) associated with IGR J18483-0311 according to Stephen et al. (2006). This, together with the huge absorption of IGR J18483-0311 as measured with INTEGRAL and Swift XRT, casts some doubt on a possible association between the ROSAT HRI source and IGR J18483-0311.

Figure 16 shows the $0.2-10 \mathrm{keV}$ light curve of the two Swift XRT observations, OBS1 and OBS2 (bin time $200 \mathrm{~s}$ ). The time axis is in seconds, counted from the beginning of OBS1, and the flux axis is in count/s. We can note a variability since the source during OBS2 is slightly brighter than OBS1. In fact, the average absorbed fluxes during OBS2 and OBS1 are $2.2 \times 10^{-11}(1-7 \mathrm{keV})$ and $4.3 \times 10^{-12}(1-7 \mathrm{keV})$, respectively. It seems that in both periods the source was not in outburst but in a low state. Fine time resolution light curves of both Swift XRT observations were searched for pulsations using the LombScargle method but no positive results were obtained; it is likely the statistics were not good enough because of the small exposure time of the observations and the low state of the source.

It is worth pointing out that the two Swift observations occured several days before the closest expected outburst of IGR J18483-0311, based upon the 18.52 days periodicity (see Table 4). Assuming that the Swift source is associated with IGR J18483-0311, the Swift XRT detections probably represent its quiescence emission.

However, we cannot entirely exclude that the Swift source is not associated with IGR J18483-0311 because of the lack of a simultaneous Swift-INTEGRAL detection during an outburst. 
Table 4. Summary of Swift XRT observations of IGR J18483-0311.

\begin{tabular}{cccccc}
\hline $\begin{array}{c}\text { Obs date } \\
\text { (MJD) }\end{array}$ & $\begin{array}{c}\text { Closest expected outburst } \\
\text { (MJD) }\end{array}$ & $\begin{array}{c}\text { Average absorbed flux } \\
\left(\mathrm{erg} \mathrm{cm}^{-2} \mathrm{~s}^{-1}\right)\end{array}$ & $\begin{array}{c}\text { Average luminosity } \\
\left(\mathrm{erg} \mathrm{s}^{-1}\right)\end{array}$ & $\Gamma$ & $\begin{array}{c}N_{\mathrm{H}} \\
\left(\mathrm{cm}^{-2}\right)\end{array}$ \\
\hline 53782 & $\sim 53788.64$ & $\sim 4.3 \times 10^{-12}(1-7 \mathrm{keV})$ & $\sim 1.67 \times 10^{34}$ & $1.7 \pm 0.7$ & $6.3_{-1.8}^{+2.4} \times 10^{22}$ \\
53799 & $\sim 53807.16$ & $\sim 2.2 \times 10^{-11}(1-7 \mathrm{keV})$ & $\sim 8.55 \times 10^{34}$ & $1.3 \pm 0.25$ & $4.8 \pm 0.9 \times 10^{22}$ \\
\hline
\end{tabular}

^ Assuming a distance of $\sim 5.7 \mathrm{kpc}$ (see Sect. 5).

\section{An highly redenned star as optical counterpart of IGR J18483-0311}

As we can note in Fig. 17, the Swift XRT very accurate position of IGR J18483-0311 (3'.3 error radius) allows us to identify an optical USNO-B1.0 source as the likely optical counterpart. The position of this star $\left(\mathrm{RA}=18^{\mathrm{h}} 48^{\mathrm{m}} 17.2^{\mathrm{s}}\right.$, Dec $\left.=-03^{\circ} 10^{\prime} 16.5^{\prime \prime},(\mathrm{J} 2000)\right)$ is $1^{\prime \prime} .06$ from the Swift XRT location of IGR J18483-0311. The optical and NIR magnitudes extracted from the USNO-B1.0 and 2MASS catalogues, respectively, are $R=19.26, I=15.32, J=10.74, H=9.29$ and $K=8.46$. This indicates an extremely reddened object, and the optical/NIR color indices of this source strongly resemble those of a heavily absorbed early-type star, similar to the case of 2RXP J130159.6-635806 = IGR J13020-6359 (Chernyakova et al. 2005), identified as a HMXB by Masetti et al. (2006). Indeed, assuming the Milky Way extinction law (Cardelli et al. 1989), we find that the optical/NIR color indices are consistent with those of a late O/early B-type star (Wegner 1994) suffering from a reddening of $A_{V} \approx 13 \mathrm{mag}$. This, using the formula of Predehl \& Schmitt (1995), implies a column density of $N_{\mathrm{H}} \sim 2.3 \times 10^{22} \mathrm{~cm}^{-2}$, which is lower than that inferred with the Swift and INTEGRAL spectral analysis results. This may indicate that part of the absorption detected in X-rays is local to the accreting object. Admittedly, if one considers the $R-I$ color index alone, one gets a higher $V$-band optical extinction ( $A_{V} \approx 16 \mathrm{mag}$ ). However, it should be noted that, for faint $(>18)$ magnitudes, the photometry of USNO catalogues can have uncertainties as high as $1 \mathrm{mag}$ or more (e.g. Masetti et al. 2003). Thus, we consider the $A_{V}$ estimate obtained using the IJHK magnitudes as a more correct one. Assuming then $A_{V} \approx 13 \mathrm{mag}$ along the IGR J18483-0311 line of sight and a B0 spectral type for the companion star in this system, we can infer its distance, depending on the luminosity class (main sequence, giant or supergiant) of the star. We find that, for these three cases, a distance of $\sim 2.1, \sim 3.5$ and $\sim 5.7 \mathrm{kpc}$, respectively, is found. The first (main sequence) case would place the system in the Sagittarius arm of the Galaxy (e.g. Leitch \& Vasisht 1998): this distance is however too close to justify the inferred optical absorption.

We consider it more likely that the secondary star is an early giant or supergiant: in this case the source would lie either in the near or in the far side of the Scutum-Crux arm, respectively. However, only optical/NIR spectroscopy of this object can help us to shed more light on its nature.

\section{Discussions and conclusions}

We report on 5 newly discovered outbursts from IGR J18483-0311 detected by IBIS. In each case the outburst activity lasts no longer that a few days and it is characterized by several fast flares with timescales of a few hours. The broad band (3-50 keV) JEM-X/ISGRI spectrum of outburst No. 1 is best fitted by an absorbed cutoff power law with $\Gamma=1.4 \pm 0.3$, $E_{\mathrm{c}}=22_{-4.5}^{+7.5} \mathrm{keV}$ and $N_{\mathrm{H}}=9_{-4}^{+5} \times 10^{22} \mathrm{~cm}^{-2}$. The latter exceeds

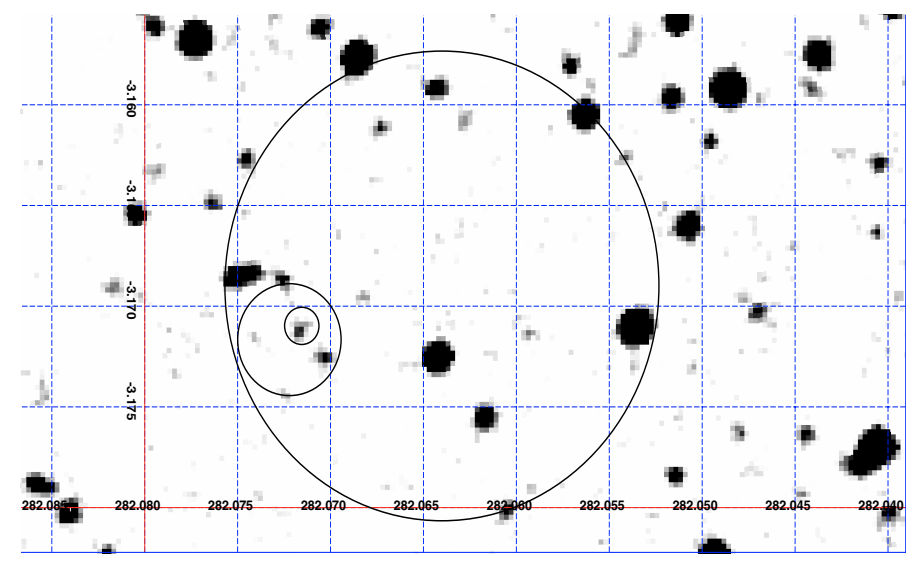

Fig. 17. USNO B1.0 optical field image (R2 magnitude). The circles represent, from larger to smaller, the ISGRI error circle of IGR J18483-0311 (Bird et al. 2006), ROSAT (Stephen et al. 2006) and Swift (this paper). As we can note, the accurate Swift position allow us to identify the likely optical counterpart for IGR J18483-0311.

the galactic absorption along the line of sight $\left(1.6 \times 10^{22} \mathrm{~cm}^{-2}\right)$ suggesting that most of the low energy absorption is intrinsic to the source.

Timing analysis performed on the long term ISGRI light curve allows us to identify a periodicity of $18.52 \pm 0.01$ days which is most likely a measurement of the orbital period of the system. Analysis of the 4-20 keV JEM-X light curve of the brightest complete outburst (No. 1 in Table 1) identifies a periodicity of $21.0526 \pm 0.0005$ seconds which could be due to the spin period of an X-ray pulsar.

From Swift XRT data analysis, we obtain a very accurate source position which finally allows us to pinpoint a single optical object as the probable counterpart of IGR J18483-0311. Its optical/NIR magnitudes indicate it as an extremely reddened object. In particular the optical/NIR color indices strongly resemble those of a heavily absorbed early-type star.

The X-ray spectral shape, the periodicities of 18.52 days and $21.0526 \mathrm{~s}$, the high intrinsic absorption, the location in the direction of the Scutum spiral arm and the highly reddened optical object as possible counterpart, support the hypothesis that IGR J18483-0311 is a HMXB with a neutron star as compact companion. Assuming the 18.52 days periodicity as the system orbital period and the 21.0526 s periodicity as the neutron star spin period, then the source lies in the Be HMXB transients region of the Corbet diagram (Corbet 1986). Moreover, the characteristics of the outbursts and their regular recurrence at $\sim 18.52$ days strongly support a Be/X-ray transient HMXB nature. However, we can not entirely exclude the source to be a supergiant fast X-ray transient because of the timing and spectral X-ray properties resembling those of already known SFXTs (Sguera et al. 2005, 2006, 2007). The typical duration of the outburst activity of IGR J18483-0311 is a few days, somewhat longer than typical outbursts from SFXTs which are usually 
shorter than a day, typically a few hours (Sguera et al. 2005, 2006). However, outbursts from SFXTs lasting a few days have been already reported (Sidoli et al. 2006). It is worth bearing in mind that SFXTs are characterized by typical luminosity ratios $L_{\text {Max }} / L_{\text {Min }} \sim 10^{4}$. Assuming that the Swift XRT detection is associated with IGR J18483-0311 and it represents its quiescence emission, then its luminosity ratio is $L_{\mathrm{Max}} / L_{\mathrm{Min}} \sim 10^{3}$, an order of magnitude smaller than that typical of SFXTs.

Spectroscopy of the optical/NIR counterpart of IGR J18483-0311 is thus essential to fully characterize this hard X-ray emitting object.

Acknowledgements. We thank the anonymous referee for very useful comments which helped us to improve the paper. This research has been supported by University of Southampton School of Physics and Astronomy. The italian coauthors acknowledge support via contract ASI/INAF I/023/05.

\section{References}

Bird, A. J., Barlow, E. J., Bassani, L., et al. 2006, ApJ, 636, 765 Cardelli, J. A., Clayton, G. C., \& Mathis, J. S. 1989, ApJ, 345, 245

Chernyakova, M, Lutovinov, A., \& Capitanio, F. 2003, ATEL 157

Chernyakova, M., Lutovinov, A., Rodríguez, J., \& Revnivtsev, M. 2005, MNRAS, 364, 455

Corbet, R. H. D. 1986, MNRAS, 220, 1047

Dean, A. J., Bazzano, A., Hill, A. B., et al. 2005, A\&A, 443, 485

Gehrels, N., Chincarini G., Giommi P., et al. 2004, ApJ, 611, 1005
Hill, A. B. 2006, Ph.D. Thesis, School of Physics and Astronomy, University of Southampton, UK

Hill, A. B., Walter, R., \& Knigge, C. 2005, A\&A, 439, 255

Horne, J. H., \& Baliunas, S. L. 1986, ApJ, 302, 757

Lebrun, F., Leray, J. P., Lavocat, P., et al. 2003, A\&A, 411, L141

Leitch, E. M., \& Vasisht, G. 1998, New Astron., 3, 51

Lund, N., Butz-Jorgentsen, C., Westergaard, N. J., et al. 2003, A\&A, 411, L231

Masetti, N., Palazzi, E., Pian, E., et al. 2003, A\&A, 404, 465

Masetti, N., Morelli, L., Palazzi, E., et al. 2006a, A\&A, 459, 21

Masetti, N., Pretorius, M. L., Palazzi, E., et al. 2006b, A\&A, 449, 1139

Molkov, S., Lutovinov, A., \& Grebenev, S. 2003a, A\&A, 411, L357

Molkov, S., Cherepashchuk, A. M., Lutovinov, A., et al. 2003b, [arXiv:astro-ph 0402416]

Monet, D. G., Levine, S. E., Canzian, B., et al. 2003, AJ, 125, 984

Moretti A., Campana S., Tagliaferri G., et al. 2004, SPIE, 5165, 232

Moretti, A., Perri, M., Capalbi, M., et al. 2006, A\&A, 448, L9

Negueruela, I., Smith, D. M., Reig, P., et al. 2005, ESA SP-604, 165

Predehl, P., \& Schmitt, J. H. M. M. 1995, A\&A, 293, 889

Press, W. H., \& Rybicki, G. B. 1989, ApJ, 338, 277P

Scargle, J. D. 1982, ApJ, 263, 835

Sguera, V., Barlow, E. J., Bird, A. J., et al. 2005, A\&A, 444, 221

Sguera, V., Bazzano, A., Bird, A. J., et al. 2006, ApJ, 646, 452

Sguera, V., Bird, A. J., Dean, A. J., et al. 2007, A\&A, 462, 695

Sidoli, L., Paizis, A., \& Mereghetti, S. 2006, A\&A, 450, L9

Skrutskie, M. F., Cutri, R. M., Stiening, R., et al. 2006, AJ, 131, 1163

Stephen, J. B., Bassani, L., Malizia, A., et al. 2006, A\&A, 445, 869

Ubertini P., Lebrun, F., Di Cocco, G., et al. 2003, A\&A, 411, L131

Walter, R., Zurita Heras, J., Bassani, L., et al. 2006, A\&A, 453, 133

Wegner, W. 1994, MNRAS, 270, 229 